\title{
On the structure of field-aligned currents in the mid-altitude cusp
}

\author{
A. Marchaudon ${ }^{1,2}$, J.-C. Cerisier ${ }^{3}$, J.-M. Bosqued ${ }^{4}$, C. J. Owen ${ }^{1}$, A. N. Fazakerley ${ }^{1}$, and A. D. Lahiff ${ }^{1}$ \\ ${ }^{1}$ Mullard Space Science Laboratory, University College London, Holmbury St Mary, Dorking, RH5 6NT, Surrey, UK \\ ${ }^{2}$ Now at: Laboratoire de Physique et Chimie de l'Environnement, 3A avenue de la Recherche Scientifique, 45071 Orléans \\ Cedex 2, France \\ ${ }^{3}$ Université Pierre et Marie Curie-Paris6; Centre d'Etude des Environnements Terrestre et Planétaires, 4 avenue de Neptune, \\ 94107 Saint-Maur-des-Fossés Cedex, France \\ ${ }^{4}$ Centre d'Etude Spatiale des Rayonnements, 9 avenue du Colonel Roche, 31028 Toulouse Cedex 4, France
}

Received: 7 September 2006 - Accepted: 26 October 2006 - Published: 21 December 2006

\begin{abstract}
We analyse two crossings of the polar cusp at midaltitudes $\left(\approx 4 R_{E}\right)$ by Cluster in order to study the structure of field-aligned currents associated with the injection of magnetosheath plasma. The current density is deduced independently from magnetic field and from particle flux measurements. In both cases the data are carefully tested. Magnetic fluctuations are analysed by discriminating between those compatible with the plane current sheet hypothesis under which the current density can be calculated safely, and those resulting from filamentary current structures. At medium transverse scales $(80 \mathrm{~km})$, the structure of the currents is more often tube-like than sheet-like, and current sheets are not systematically elongated in the east-west direction. The total particle current is calculated from the electron and ion measurements. For electrons, the full energy range is taken into account, from above the photoelectron threshold up to $32 \mathrm{keV}$. Magnetosheath plasma injections are well correlated with pairs of field-aligned currents. In both cases, the parallel current is mainly carried by electrons while ions contribute for about $20 \%$. In the plane current sheets, the ratio between magnetic and particle currents shows large variations between 0.4 and 1.1. These fluctuations can be explained by the convective motion of the current sheets.
\end{abstract}

Keywords. Magnetospheric physics (Current systems; Magnetopause, cusp and boundary layers; Solar windmagnetosphere interactions)

\section{Introduction}

The large-scale distribution of field-aligned currents (FACs) connecting the Earth magnetosphere and ionosphere consists of two concentric zones encircling the magnetic pole (Iijima and Potemra, 1976). Currents flow in opposite di-

Correspondence to: A. Marchaudon

(aurelie.marchaudon@cnrs-orleans.fr) rection in the two zones and also on each side of the noonmidnight plane. The low-latitude currents, called Region-2, are mainly due to pressure gradients in the inner magnetosphere, while the high-latitude currents, called Region-1, flow at the interface between open and closed field lines and are due to the interaction between the solar wind and the magnetosphere. Region-2 maps into the Central Plasma Sheet (CPS) and Region-1 maps in the Plasma Sheet Boundary Layer and Low-Latitude Boundary Layer (PSBL/LLBL). The particle carriers in the large-scale Region-2/Region-1 FACs have been extensively studied in the dawn and dusk sectors (Klumpar, 1979; Sugiura et al., 1984; Yamauchi et al., 1998). In the dusk sector, downward Region-2 currents are carried by cold electrons extracted from the ionosphere and upward Region- 1 currents are essentially carried by precipitating electrons from the PSBL/LLBL. In the dawn sector, upward Region- 2 currents are carried by precipitating electrons from the CPS and downward Region-1 currents are carried by upward ionospheric electron bursts.

The systems of currents in the midnight and noon sectors are more complicated. The nightside pattern depends upon substorm activity while the dayside pattern depends upon interplanetary magnetic field (IMF) conditions. Statistical studies by Erlandson et al. (1988) during southward IMF and Iijima et al. (1984) during northward IMF showed that the large-scale dayside distribution of FACs depends strongly upon the north-south and dawn-dusk components of the IMF. In addition, meso-scale FACs due to direct interaction of the solar wind with the magnetosphere e.g. via sporadic reconnection forming flux transfer events (Southwood, 1987; Escoubet et al., 1992; Marchaudon et al., 2004), dominate and are superimposed on the large-scale FACs. The relation between meso-scale and large-scale FACs, both in terms of current density and in terms of current carriers remain a subject of debate, although they have been extensively discussed by Potemra et al. (1977), McDiarmid et al. (1978), Yamauchi et al. (1998), and Oksavik et al. (2004).

Published by Copernicus GmbH on behalf of the European Geosciences Union. 
Only a few papers have presented quantitative comparisons between field-aligned currents deduced from magnetic and from charged particle measurements. Attempts have been made in the dayside by Berko et al. (1975) and Burch et al. (1983), and in the nightside by Klumpar et al. (1976), Theile and Wilhelm (1980), Sugiura et al. (1984), Burke (1984) and Hoffman et al. (1985). In most of these studies, a comparison of field-aligned current profiles deduced from the two methods was presented. The results were not convincing, either in amplitude or in direction. In particular, downward currents were often not observed in particle data. The suggested explanation was the incomplete energy coverage of the particle detectors at low-energy. Burch et al. (1983) presented one of the best comparisons using data from the DE-1 satellite in the polar cusp. These authors explained that because the cold ionospheric electrons carrying the downward currents were accelerated upward by a potential drop, they could only be detected onboard the spacecraft when it was situated above the potential drop. However, the magnetic and particle current profiles shown were far from identical, especially in the downward current regions. More recently, based on FAST satellite measurements, Elphic et al. (1998) claimed to have obtained a "fairly good" agreement between the current densities calculated from the electrons fluxes alone and from the magnetic data, but no profile of the two determinations were presented.

The determination of FACs from magnetic field measurements onboard a single spacecraft is also not free from criticism. An infinite current sheet structure is usually assumed, often with an east-west orientation of the sheet. While these assumptions have proved to be valid and have been widely used when describing the larger-scale parallel currents (Potemra, 1985), smaller-scale magnetic variations often do not fit this infinite current sheet model, thus revealing a more complex filamentary structure. Several attempts have been made to model the effects of finite size currents sheets (Fung and Hoffman, 1992; Seran and Cerisier, 2005) or to interpret magnetic variations in terms of current tubes (Robert et al., 1984; Berthelier et al., 1988). However, because the underlying models depend upon a too large number of parameters, these methods do not lead to an unambiguous determination of the current density and structure.

This paper represents an attempt to study meso-scale parallel currents observed in the polar cusp based on simultaneous particle and magnetic field measurements. To interpret magnetic data, we have developed simple tests to select situations where the infinite current sheet hypothesis is valid. We have used magnetic, electron and ion data from the Cluster satellites, taking advantage of the good coverage of the electron experiment in the lower energy range. Two events, during two cusp crossings (29 August 2002 and 10 September 2002) are studied, during which Cluster flew from the dayside to the nightside at mid-altitude $\left(4-5 R_{E}\right)$, in the 12.2-12.6 MLT sector. The data from each spacecraft were studied independently and based on their completeness, their quality (high resolution data) and consistency, only the best data sets were selected for detailed analysis. The results are compared qualitatively and quantitatively in terms of the various sources of discrepancy between the particle and magnetic currents and their implication on the current structure is discussed.

\section{Instrumentation}

Cluster consists in four identical satellites on an elliptical polar orbit with a perigee of $\sim 4 R_{E}$, an apogee of $\sim 19 R_{E}$ and a period of $\sim 58 \mathrm{~h}$. The events presented in this study occur during summer 2002, when Cluster crosses the northern midaltitude cusp near perigee in the burst mode. Electron, ion and magnetic field data are used. The four Cluster spacecraft are in an approximately co-linear formation, which prevents an accurate evaluation of the current density by the curlometer technique.

The Plasma Electron and Current Experiment (PEACE) (Johnstone et al., 1997) provides the electron velocity distribution function every $4 \mathrm{~s}$ (spacecraft spin period), in the energy range from $\sim 1 \mathrm{eV}$ to $\sim 26 \mathrm{keV}$. PEACE is composed of a Low-Energy Electron Analyser (LEEA) and a High Energy Electron Analyser (HEEA) located on opposite sides of the spacecraft, which measure overlapping energy ranges. In burst mode, the full three-dimensional (3-D) distribution is available and the resulting density and velocity moments are calculated on the ground with a resolution up to $4 \mathrm{~s}$. The energy range falling below the spacecraft potential $U_{s c}$ is first removed in order to eliminate the photoelectrons from the moment calculations. The remaining energy range is then rescaled to remove the electron acceleration due to the spacecraft potential.

The Cluster Ion Spectrometry (CIS) experiment consists of two different instruments: the Hot Ion Analyser (HIA) and the COmposition and DIstribution Function analyser (CODIF) (Rème et al., 2001). Both instruments were switched off on sc-2 and HIA was also switched off on sc4. For this study, we use HIA on sc-1 which offers a good energy and angular resolution without mass resolution and provides a full three-dimensional energy/velocity distribution (assuming all ions are protons) from thermal energies up to about $32 \mathrm{keV} / \mathrm{q}$. Since HIA is not available on sc-4, we use CODIF which offers a medium angular resolution but measures the full three-dimensional energy/velocity distribution of the major magnetospheric ions $\left(\mathrm{H}^{+}, \mathrm{He}^{+}, \mathrm{He}^{++}\right.$and $\left.\mathrm{O}^{+}\right)$ up to about $38 \mathrm{keV} / \mathrm{q}$. Only the proton distribution is used in this case. The density and velocity moments are calculated onboard with a $4 \mathrm{~s}$ resolution.

The Flux Gate Magnetometer (FGM) (Balogh et al., 2001) measures the 3-D magnetic field vector, with up to $\sim 202$ vectors/s sampling. In this paper, we use data averaged to $4 \mathrm{~s}$ resolution. 
Interplanetary magnetic field and solar wind data from the ACE satellite are also used for monitoring the interplanetary medium, with an appropriate lag to take into account the solar wind propagation from ACE to Earth.

\section{Analysis of magnetic variations in terms of field- aligned currents}

In the magnetosphere where perpendicular currents are negligible, the field-aligned current density is related to magnetic variations by Ampere's law

$\mathbf{J}_{/ /}=\frac{\nabla \times \mathbf{b}}{\mu_{o}}$

where $\mathbf{b}$ is the perturbation magnetic field obtained after removing remote sources such as the Earths main field and the field arising from non-local currents. Since $\nabla \times \mathbf{b}$ cannot be calculated from the magnetic perturbations measured along a single satellite trajectory, it is usually assumed that the currents are distributed in infinite parallel current sheets, without even testing the validity of this model. Large errors in the current density may then result. For instance, applying an infinite current sheet algorithm to measurements made in the vicinity, but outside of a current tube (i.e. in a region where no current flows) returns a result suggesting a finite current density at that point in space. Also, the same analysis applied to a crossing of the tube itself will return a changing sheet normal direction, except in the particular case of a central crossing. It is thus important to be able to identify the situations when the infinite current sheet hypothesis is valid, in order to validate the calculated current density. This is crucial if one wants to make fully quantitative comparisons with particle measurements.

For this purpose, we have modelled the magnetic perturbation created along a satellite orbit by two extreme parallel current structures: a circular current tube and a pair of opposite parallel infinite current sheets. The orbit is in the plane perpendicular to the ambient magnetic field. Panels (a) and (b) of Fig. 1 display the two components of the magnetic field perturbation in the plane perpendicular to the ambient field, measured along the orbit. The left panels are for the parallel current sheets crossed at an angle of $30^{\circ}$. The right panels are for a tube tangent to the orbit. In both cases, we have calculated the covariance matrix of the magnetic signal and its eigenvalues and eigenvectors. Panels (c) and (d) show the results obtained for two parameters which appear to be the most pertinent to discriminate between the two structures: (1) the angle $\alpha$ (measured in the plane perpendicular to the ambient field from a fixed reference axis, here the direction of the orbit) defining the direction of the eigenvector associated with the largest eigenvalue (panels c) and (2) the ratio $r$ between the intermediate and the largest eigenvalues (panels d). In the following, these two parameters will be called "polarisation angle" for $\alpha$ and "variance ratio" for $r$.

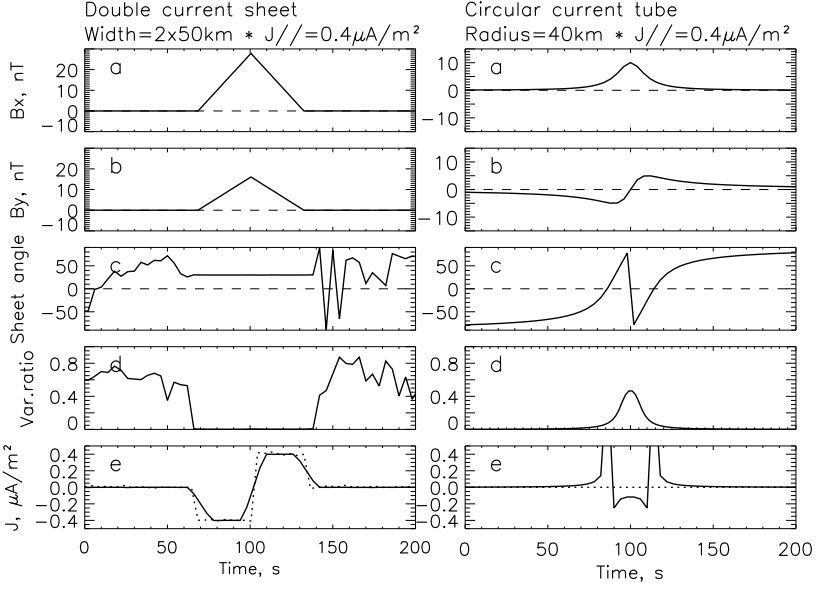

Fig. 1. Models of the magnetic perturbation created by a pair of opposite parallel infinite current sheets (left) and a circular current tube (right). From top to bottom: (a) and (b) the two components of the magnetic field perturbation in the plane perpendicular to the current direction; (c) the angle defining the direction of the eigenvector associated with the largest eigenvalue; (d) the ratio between the intermediate and the largest eigenvalues; (e) the current density $($ modelled $=$ dotted line; obtained by applying the infinite sheet hypothesis = solid line).

In the absence of noise, the variance ratio $r$ is equal to zero and the polarisation angle $\alpha$ remains constant when crossing the infinite current sheets system. Conversely, $r$ remains strictly positive and $\alpha$ shows a systematic oscillation when the satellite passes close to the current tube. These different behaviours result from the magnetic field structure, linearly polarized across parallel current sheets and rotating around a current tube. Of course, $r$ and $\alpha$ show random variations in regions of zero current (outside the current sheets). Finally, panels (e) of Fig. 1 show the modelled current density (dotted line) and the current density deduced from the magnetic perturbations (solid line), applying the infinite sheet hypothesis (method described in more details below). As expected the true current density is retrieved in the double sheet case, with only some smoothing at the edges of the sheets. Conversely, the current profile obtained in the tube case does not show any clear relation with the true one. Note that the numerical values (current density $=0.4 \mu \mathrm{A} . \mathrm{m}^{-2}$, size of current structures $=40 \mathrm{~km}$, satellite velocity $=4 \mathrm{~km} . \mathrm{s}^{-1}$, sampling frequency $=0.25 \mathrm{~s}^{-1}$ ) used for modelling the results of Fig. 1 are typical of those encountered in our Cluster cusp data analysis. The covariance matrix is calculated over 5 points, which results from a compromise between opposite objectives, namely resolving small-scale currents and obtaining meaningful eigenvectors and eigenvalues. Varying the physical parameters of the current structure by a factor of 2 does not change the above qualitative conclusions, neither does the introduction of noise ( $1 \mathrm{nT}$ r.m.s.) in the modelled data. We conclude that in the analysis of real data, we can 
assume that we are dealing with current sheets only when both the variance ratio $r$ is small and the polarisation angle $\alpha$ is stable. If one at least of these conditions is not verified, the local current density cannot be determined safely.

The Cluster satellite data have been processed as follows. In order to remove the background field and to keep only the perturbation field $\mathbf{b}$ due to the local currents, we have chosen to detrend the magnetic field by subtracting a polynomial fit calculated on two time intervals ( $\sim 3-5 \mathrm{~min})$ immediately before and after crossing the cusp injections where the largest magnetic variations are observed. The perturbation field $\mathbf{b}$ is then separated into its component $\mathbf{b}_{/ /}$parallel to the mean field, representing the diamagnetic plasma effect, and its perpendicular component $\mathbf{b}_{\perp}$ due to the field-aligned currents. As in the above modelling, the current sheet orientation in the plane perpendicular to the main field is given by the direction of the eigenvector associated with the largest eigenvalue of the covariance matrix of $\mathbf{b}_{\perp}$, while the sheet normal is given by the intermediate eigenvalue. Applying the static current sheet model (which excludes the possibility of Alfvén waves), the curl in Eq. (1) reduces to the time derivative of the component of $\mathbf{b}_{\perp}$ in the sheet direction. Choosing a Cartesian reference frame where the $z$ axis is along the mean magnetic field and the $x$ and $y$ axis are respectively parallel to the sheet direction (the direction of maximum variance) and normal to the sheet plane (the direction of intermediate variance), the current density is given by

$J_{/ /}=\frac{1}{\mu_{0}} \frac{\partial b_{x}}{\partial t} \frac{1}{v_{n}}$

where $v_{n}$ is the projection of the spacecraft velocity along the $y$ axis (the normal to the sheet plane). Positive $J_{/ /}$corresponds to an upward current in the northern hemisphere. The current density becomes infinite and the method fails when the spacecraft velocity is parallel to the current sheet (the $x z$ plane).

In case of a drift of the current sheet, the velocity $v_{n}$ in Eq. (2) is the velocity of the observer relative to the sheet $\left(v_{\text {spacescraft }}-v_{\text {sheet }}\right)_{n}$. Lockwood et al. (2001) have shown that the sheet velocity may be of the same order as the spacecraft velocity near Cluster perigee at medium altitude in the magnetosphere. In the absence of any reliable evaluation of the sheet velocity, we have assumed stationary current sheets. The consequences will be discussed later in Sect. 6.4.

\section{Determination of the particle currents}

The particle field-aligned current is the sum of the electron and ion currents obtained from the electron and ion density and velocity component parallel to the magnetic field direction

$J_{/ / \text {part }}=J_{/ / e}+J_{/ / i}=-e n_{e} V_{/ / e}+e n_{i} V_{/ / i}$ with positive $J_{/ / i, e}$ and $V_{/ / i, e}$ corresponding to upward currents and velocities, $n_{i, e}$ corresponding to densities and $e$ the Coulomb charge.

\subsection{Uncertainties in particle current determination}

When calculating the electron moments, the contribution of photoelectrons has to be removed. As moments are calculated on the ground using burst mode 3-D distribution data, the variable spacecraft potential, deduced from the electric field experiment, is used and the upper limit of rejected energy bins checked visually. The estimated precision of this ground processing (defined as the sum of the photoelectron processing and a comparison between the overlapping energy range of the low and high energy sensors) is 10\% for the density and 20-25\% for the velocity. Moreover, the PEACE and WHISPER electron densities have been compared. The WHISPER density, deduced from the electron plasma frequency, is not subject to the errors associated with the photoelectron population, thus providing an independent estimate of the precision of the PEACE-derived measurement of the ambient electron density. The discrepancy between these two electron densities can amount to $15 \%$, slightly higher than the above PEACE estimate of $10 \%$. Summing the density and velocity errors leads to a maximum error on the electron current of $\sim 35-40 \%$. Concerning ion moment processing, the density error is $5 \%$ and the velocity error is $5-10 \%$. The CIS and WHISPER densities have also been compared, assuming quasi-neutrality. The discrepancy between the two densities can reach $35-40 \%$, which can be attributed to calibration errors or saturation in the ion detector (especially CODIF) when the density is large. The maximal error on the ion current (sum of the density and velocity errors) is then of $\sim 40-50 \%$.

The uncertainty on the total current is thus $35-50 \%$ when both the electron and ion currents have the same sign or when the ion current is small. Otherwise the uncertainty may be larger.

\subsection{Aliasing effect on particle velocity}

The error on the velocity deduced from particle distribution can be strongly increased inside sharp density gradients by aliasing effects. For particle instruments onboard a spinning spacecraft, it is usual to measure the full 3-D distribution as the instrument aperture rotates with the spacecraft. Thus all look-directions are not observed simultaneously and a full spin period is needed before the moments of the particle distribution function can be calculated. When a spinning spacecraft flies across a density gradient, and experiences variations in the particle distribution which are fast compared to the spin period, an additional, artificial component in the calculated flow velocity perpendicular to the spin axis, may be introduced. 
The CIS experiment (either HIA or CODIF) is composed of only one sensor and the aliasing effect on the ion velocity is difficult to detect. This effect is however more readily detectable in the electron velocity since the PEACE experiment is composed of two sensors (LEEA and HEEA) located on opposite sides of the spacecraft. During a full spin period, the two sensors will observe the same direction of arrival with a half-spin delay $(\sim 2 \mathrm{~s})$. If a sharp density gradient is crossed during the spin, a same given direction of arrival will be observed in different plasmas by the two sensors, and an apparent flow in opposite directions between the two sensors may then be generated. When the additional flow velocity is large, the components of the velocity lying in the plane perpendicular to the spin axis (usually $V_{x}$ and $V_{y}$ in GSE coordinates) are opposite in sign between the 2 electron sensors. In this paper, we remove the electron aliasing effect by averaging the moments given by the two PEACE sensors.

\section{Cluster observations of the field-aligned currents}

We study two mid-altitude northern cusp crossings (29 August 2002 and 10 September 2002) by the Cluster spacecraft, concentrating on the spacecraft for which ion, electron and magnetic data are available with clear signatures of fieldaligned currents. We study the cusp data of sc-1 for $29 \mathrm{Au}-$ gust 2002 and sc-4 for 10 September 2002. In the rest of the paper, we refer to these events as: event A for sc-1 (29 August 2002) and event B for sc-4 (10 September 2002). The two data sets are plotted in the same format in Figs. 2 and 3 , with, from top to bottom, CIS ion spectrogram in the direction parallel (downward) to the magnetic field (panels a), PEACE electron spectrogram in the parallel and anti-parallel directions (panels $b, c$ ), electron and ion field-aligned currents at the resolution of the particle distributions (panels d). Panels (e) show the components perpendicular and parallel to the local magnetic field of the FGM magnetic perturbations. The perpendicular component of the perturbation is directly associated with the field-aligned currents and the parallel component is a measure of the diamagnetic effect due to particle injection. The next two panels display the results of the variance analysis of the perpendicular magnetic perturbation. The polarisation angle (panel f), defining the direction of the eigenvector associated with the largest eigenvalue, is now measured from the magnetic east, providing in addition a physically meaningful direction of the current sheet. Panel (g) shows the variance ratio between the two largest eigenvalues. Finally, the total particle and magnetic fieldaligned currents are shown on panels (h). The FACs deduced from magnetic data are calculated over 5 points $(\sim 20 \mathrm{~s})$ with $v_{\text {sheet }}=0$ only, and are not defined where the angle between the current sheet and the spacecraft trajectory is less than $5^{\circ}$. The FACs deduced from particle data are smoothed over 3 or 5 points $(\sim 20-24 \mathrm{~s})$ depending on the moment resolution, for better comparison with magnetic FACs.

\subsection{Event A, sc-1 (29 August 2002 - 16:06-16:15 UT)}

Cluster results during event A are displayed in Fig. 2. During this event, the IMF- $B_{z}$ component undergoes a sharp inversion from negative $(-5 \mathrm{nT})$ to positive $(+5 \mathrm{nT})$, strongly affecting the cusp configuration around 16:11 UT, while the IMF- $B_{x}$ and $-B_{y}$ exhibit only limited variations $\left(B_{x} \sim 5 \mathrm{nT}\right.$; $\left.B_{y} \sim-4 \mathrm{nT}\right)$ at this time. Prior to this inversion, between 16:08:45 and 16:09:35 UT (between the 1st and 2nd vertical dashed lines), sc-1 crosses the Low-Latitude Boundary Layer (LLBL) which is characterised by lower particle flux than in the cusp proper and displays small-scale fieldaligned electron structures (panels $2 \mathrm{a}, \mathrm{b}, \mathrm{c}$ ). Sc-1 was probably skimming this boundary layer before this time, since these electron field-aligned structures were intermittently observed from 16:06:35 UT. At 16:09:35 UT (2nd vertical dashed line), sc- 1 enters the cusp proper ( $\sim 76-78^{\circ}$ ILAT), indicated by intense ion and electron fluxes, with a mean energy of $700 \mathrm{eV}$ for the ions (panel 2a) and $100 \mathrm{eV}$ for the electrons (panels $2 b, 2 c$ ). The electron precipitation is continuous while the ion precipitation displays two main injections with relatively clear energy-time dispersions (2nd and 3 rd vertical dashed lines). These dispersions are consistent with reconnection at the dayside magnetopause, in agreement with the negative IMF- $B_{z}$ component observed by ACE before 16:11:00 UT. Indeed, newly reconnected field lines are transported anti-sunward by the magnetic tension at the reconnection site and by the solar wind flow. Since ions with decreasing energy precipitate with increasing time, dispersions are generated by the velocity filter effect due to field line convection (Reiff et al., 1977). The convection velocity deduced from ion measurements by HIA (not shown) is mainly duskward $\left(\sim 30 \mathrm{~km} . \mathrm{s}^{-1}\right)$ in the two injections, consistent with expectations based on the prevailing negative IMF$B_{y}$. At 16:10:50 UT, the second dispersed ion injection stops abruptly and the electron flux also decreases significantly in response to the reversal of the IMF- $B_{z}$ component from negative to positive. From 16:11:45 (4th vertical dashed line) to 16:15:00 UT, a new, less intense ion injection is observed and the anti-parallel electron flux becomes more structured than that in the parallel direction, with small-scale features at low energy (down to $\sim 10 \mathrm{eV}$ ). These structures are probably due to changes in the polar cusp configuration, after the IMF- $B_{z}$ inversion. Around 16:23:00 UT (not shown in Fig. 2$)$, sc- 1 crosses a new cusp at higher latitudes $\left(\sim 78-79^{\circ}\right.$ ILAT) which is probably associated with lobe reconnection caused by the newly-established positive IMF- $B_{z}$. This interpretation of Cluster particle observations in terms of temporal rather than spatial variations is confirmed by SuperDARN radar data, which show that the echoes backscattered from the cusp throat shift from 75 to $81^{\circ}$ ILAT, at the time of the IMF- $B_{z}$ inversion. The ion and electron currents show that the FACs are mainly carried by the electrons (panel 2d). The ion contribution is negligible in this event. The second injection (16:09:55-16:11:15 UT) is characterised by an intense 


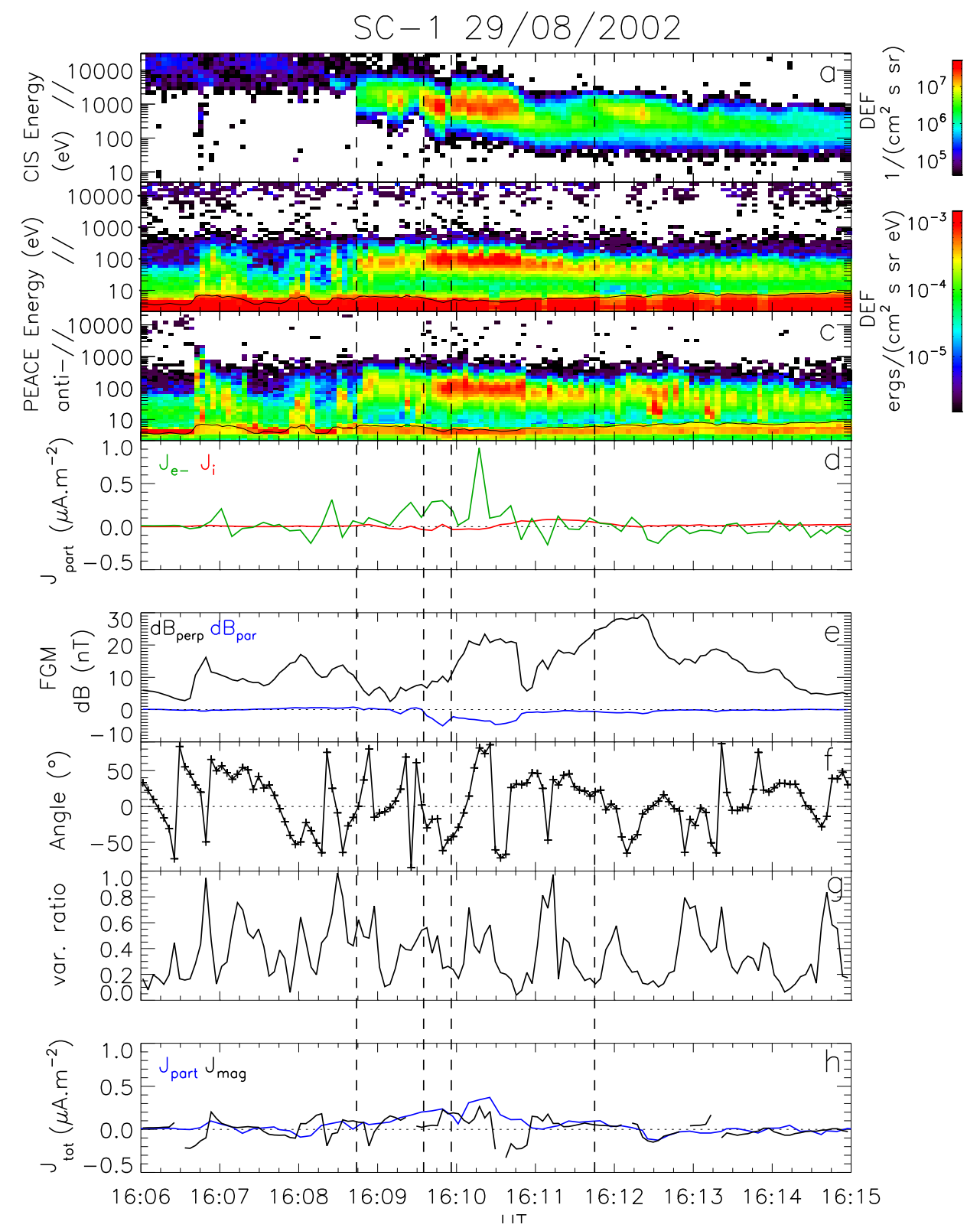

Fig. 2. Cluster data for event A (sc-1, 29 August 2002). (a) CIS ion spectrogram in the direction parallel to the magnetic field; (b), (c) PEACE electron spectrogram in the parallel and anti-parallel directions; (d) electron and ion field-aligned currents; (e) perpendicular and parallel components of the FGM magnetic field perturbation; (f) angle between the eigenvector associated with the largest eigenvalue and the local magnetic east; (g) ratio between the two largest eigenvalues; (h) total particle and magnetic field-aligned currents. The four vertical dashed lines indicate the LLBL entry, the cusp entry and the beginning of each ion injection, respectively.

upward electron field-aligned current, up to $0.9 \mu \mathrm{A} \mathrm{m}^{-2}$, followed by a smaller (in amplitude and extension) downward current.

The parallel component of the magnetic perturbation remains small except during the main particle precipitation where it decreases to about $-5 \mathrm{nT}$, showing the diamagnetic effect. The perpendicular component of the magnetic perturbation shows small-scale variations superimposed to the more global variation, revealing the presence of relatively intense small-scale FACs (panel 2e). The polarisation angle is variable between 16:08:15 and 16:10:40 UT (panel 2f), especially inside the first injection and at the beginning of the second. Several short periods display a more stable angle: between $+20^{\circ}$ and $+50^{\circ}$ (16:06:55-16:07:45 UT, 16:10:4016:11:50 UT and 16:13:55-16:14:25 UT) and around $0^{\circ}$ (16:08:55-16:09:15 UT and 16:12:20-16:12:50 UT). The 


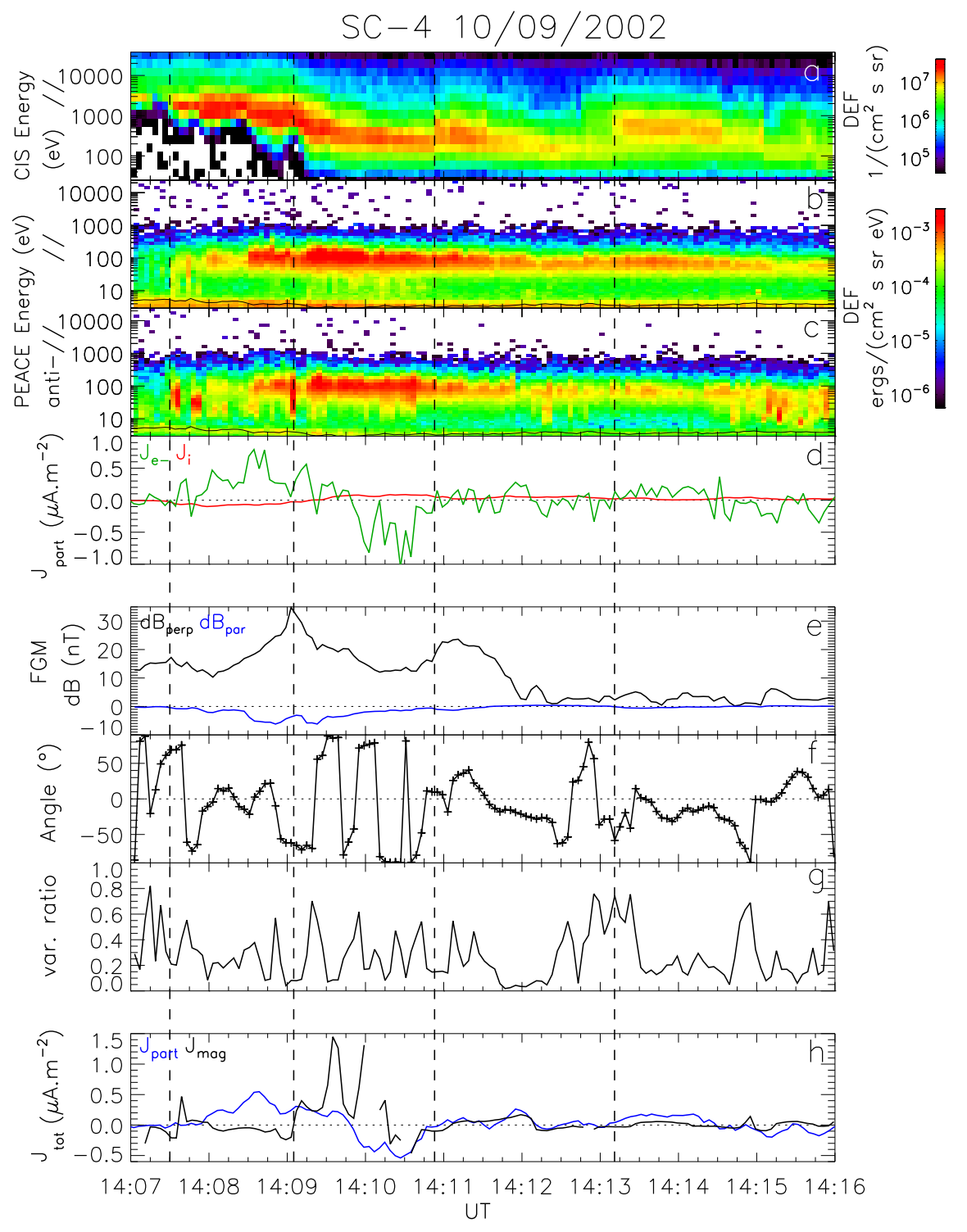

Fig. 3. Cluster data for event B (sc-4, 10 September 2002). Same format as Fig. 2.

variance ratio is also very variable during this event (panel $2 \mathrm{~g}$ ). The periods when the polarisation angle is stable show often a smaller (below 0.2) and more stable variance ratio, indicating sheet-like current structures.

For this event, the resolution of the electron moments is about $8 \mathrm{~s}$ and the total FACs deduced from particle data are smoothed over $24 \mathrm{~s}$. The FACs thus deduced from magnetic and particle data show a similar global trend (panel $2 \mathrm{~h}$ ), even if discrepancies are observed in sign between 16:08:00 and 16:08:30 UT and between 16:10:30 and 16:10:55 UT and also in amplitude between 16:09:30 and 16:10:30 UT during the main injection.
5.2 Event B, sc-4 (10 September 2002 - 14:07-14:16 UT)

Cluster results during event B are displayed in Fig. 3. Event $\mathrm{B}$ occurs during a period of stable IMF- $B_{x}$ and $-B_{z}\left(B_{x} \sim-\right.$ $6 \mathrm{nT} ; B_{z} \sim-5 \mathrm{nT}$ ) while the IMF- $B_{y}$ increases from 0.5 to $\sim 2 \mathrm{nT}$, after a slightly negative period. This IMF configuration, with a strong negative IMF- $B_{z}$, is consistent with the occurrence of merging at the dayside magnetopause. Sc-4 crosses first the LLBL between 14:07:30 and 14:08:30 UT (between the 1st and 2nd vertical dashed lines), with lower electron flux and higher ion energy than in the cusp proper (panels $3 \mathrm{a}, \mathrm{b}, \mathrm{c}$ ). Then at 14:08:30 UT (2nd vertical dashed line), sc- 4 enters the cusp region ( $76.5-78.5^{\circ}$ ILAT), where 


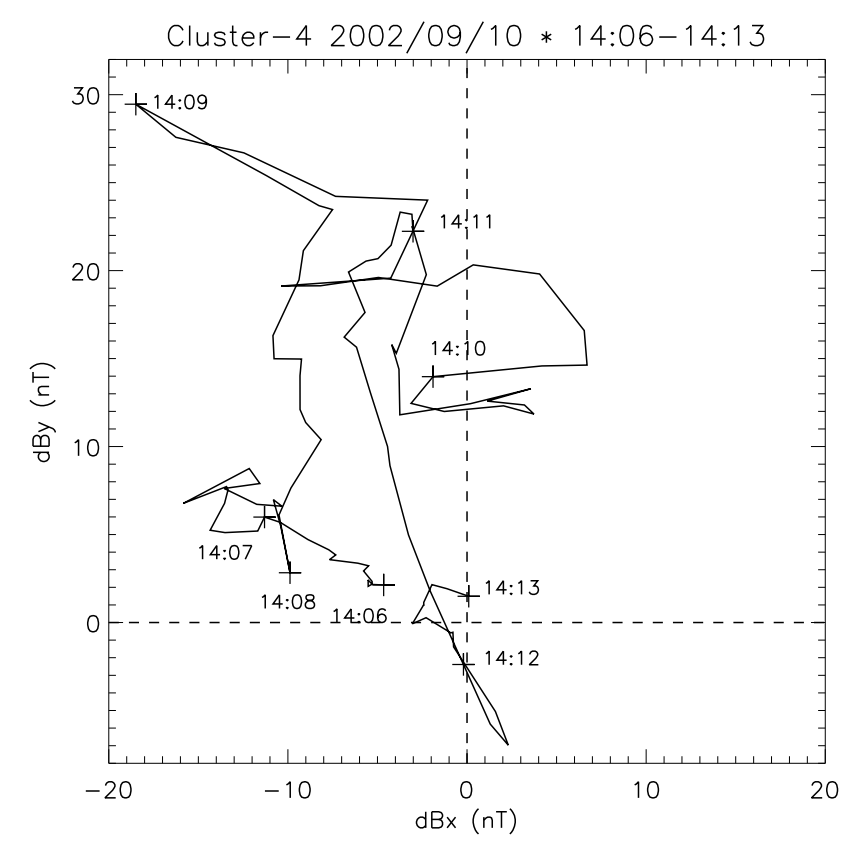

Fig. 4. Hodograph of the perpendicular component of the magnetic perturbation for the period 14:06-14:13 UT during event B (sc-4, 10 September 2002). The $x$ and $y$ directions are respectively in the magnetic meridian plane and perpendicular to it.

the mean ion energy is $500 \mathrm{eV}$ and the mean electron energy is $100 \mathrm{eV}$. Three injections (indicated by the last three vertical dashed lines) are observed in the ion spectrogram between 14:08:30 and 14:16:00 UT (panel 3a). The first two injections have relatively clear energy-time dispersion associated again with the velocity filter effect. The injections look fairly smooth in the parallel electrons (panel 3b). However, the anti-parallel electrons are more structured and show several small-scale, sporadic structures at low energy $(\sim 20-30 \mathrm{eV})$ (panel $3 \mathrm{c}$ ). Ion and electron fluxes during the third injection are less intense than during the previous ones. The CODIF convection velocity (not shown) is essentially duskward $\left(\sim 20-30 \mathrm{~km} . \mathrm{s}^{-1}\right)$ during the cusp crossing, which is unusual for positive, although small IMF- $B_{y}$. A dawnward excursion of the velocity is observed at the LLBL/cusp interface (14:07:55-14:09:05 UT). For this event, the FACs deduced from particle data are mainly carried by the electrons (panel 3d), with a negligible ion contribution. The part of the LLBL close to the cusp proper and the first cusp injection (14:08:00-14:10:50 UT) are the regions where the most intense electron field aligned-currents are observed, first upward, up to $0.8 \mu \mathrm{A} . \mathrm{m}^{-2}$, then downward.

The parallel component of the magnetic perturbation is slightly negative during the entire period and reaches a minimum during the first injection $(\sim-7 \mathrm{nT})$. The perpendicular component of the magnetic perturbation is slightly perturbed between 14:08:00 and 14:11:00 UT, giving relatively intense small-scale magnetic FACs during the first injection (panel 3e). The polarisation angle is fairly stable: around $0^{\circ}$ at the LLBL/cusp interface (14:07:55-14:08:50 UT), oscillating between $-10^{\circ}$ and $-20^{\circ}$ inside the second injection (14:11:35-14:12:20 UT), and oscillating between $-10^{\circ}$ and $-30^{\circ}$ inside the third injection (14:13:40-14:14:45 UT) (panel 3f). On the contrary, the polarisation angle is very variable during the first injection and at the interface between the second and the third injections. The variance ratio follows a similar profile, stable and below 0.2 where the angle is stable and variable and larger elsewhere, especially during the first injection (panel 3g).

For this event, the resolution of the electron moments is $4 \mathrm{~s}$ and the total FACs deduced from particle data are smoothed over 5 points $(\sim 20 \mathrm{~s})$. The magnetic and particle currents show a fairly similar profile in direction (panel $3 \mathrm{~h}$ ), except around the LLBL/cusp interface (14:07:55-14:09:05 UT) where the two currents are opposite in sign and during the first injection (14:09:25-14:10:15 UT) where the two currents show first a large discrepancy in amplitude and are then even opposite in sign. After the first injection, the magnetic and particle currents show a better agreement, even if small amplitude discrepancies still exist.

\section{Discussion}

6.1 Occurrence and polarisation of current sheets and current tubes

The analysis of the two events shows that the largest currents, either tube-like or sheet-like, occur as upward-downward pairs associated with plasma injections. The variance ratio (panels g in Figs. 2 and 3) indicates that the percentage of measurements with a ratio larger than 0.2 is $59 \%$ and $52 \%$ for events $\mathrm{A}$ and $\mathrm{B}$ respectively, indicating that tube-like structure are slightly predominant. Spatially persistent current sheets are rare. The clearest examples are the periods $16: 12: 20$ to $16: 12: 50$ UT during event A, 14:11:35 to $14: 12: 20$ UT and $14: 13: 40$ to $14: 14: 45$ UT during event B. This result may be related to the morphology of the polar cusp aurora, characterized by rayed and fragmented arcs rather than elongated and homogeneous (Sandholt et al., 2004a, b).

Figure 4 shows the hodograph of the perpendicular component of the magnetic perturbation during event $\mathrm{B}$, for the period 14:06:00-14:13:00 UT. The $x$ and $y$ directions are respectively in the magnetic meridian plane and perpendicular to it. The polarisation of the large-scale perturbation field (considered on the full 7-min period) is predominantly in the $y$ direction, indicating a longitudinal structure of the largescale current. The small-scale sheet-like structures, which can be identified by rectilinear segments in the hodograph, have a variable orientation. For instance, the direction of the double-sheet structure around 14:09:00 UT is closer to the meridian plane than longitudinal and the sheets around 
14:10:00 and 14:11:00 UT are totally along the $x$ direction. This behaviour is observed in both events, with small-scale sheets more randomly oriented than the larger ones.

\subsection{Particle carriers of the field-aligned currents}

Our quantitative evaluation of field-aligned currents from particle moments in the mid-altitude cusp, gives new insights into the particle carriers of the cusp currents. The pattern of the cusp field-aligned currents already described with the magnetic data (see Sect. 6.1), is confirmed by the particle data: they occur mostly as pairs of opposite currents associated with plasma injections in agreement with previous models and observations (Lockwood et al., 2001).

For the two events, the electron current is intense and variable, while the ion current is very small and smooth (panels d of Figs. 2 and 3). The average of the absolute value of the ion current is $20 \%$ of the electron current. Note that the ion current may be underestimated by a factor $\sim 2$ in event $B$, due to the use of the CODIF experiment which is easily subject to saturation in dense plasma regions. We observe that the electron and ion currents have opposite signs during the injections (panel 3d).

In terms of particle carriers, the upward current is carried by downward electrons from the main cusp precipitation in the $50-300 \mathrm{eV}$ energy range (panels b of Figs. 2 and 3). The downward current is carried by upward electrons, probably from ionospheric origin and distributed as small-scale and sporadic structures in the $10-40 \mathrm{eV}$ energy range, well below the main cusp precipitation (and coexisting with it) but well above the spacecraft potential, which avoids confusion with photoelectrons (panels c of Figs. 2 and 3). Most of these upward low-energy electron fluxes are associated with a downward field-aligned current detected in the net electron current as well as in the magnetic current (panels $\mathrm{d}$ and $\mathrm{h}$ of Figs. 2 and 3). This result confirms unambiguously the particle carriers (Burch et al., 1990) and suggests that, contrary to previous satellite missions (Berko et al., 1975, and Klumpar et al., 1976), the cold ionospheric electron component is not missed by the Cluster particle detectors. The ion current is carried by the main precipitation from the LLBL in the $1-4 \mathrm{keV}$ energy range and from the cusp in the $0.3-2 \mathrm{keV}$ energy range (panels a of Figs. 2 and 3).

\subsection{Comparison between the two current determinations}

For both events, the particle and magnetic currents show the same general trend. Inside the injections, discrepancies are however observed both in sign and in amplitude. Outside the injections where the FACs are smaller, the agreement is fairly good in sign, even if amplitude discrepancies of the currents remain. The agreement is particularly good during event $\mathrm{A}$ between 16:11:20 and 16:15:00 UT and for event B between 14:10:35 UT and 14:16:00 UT.
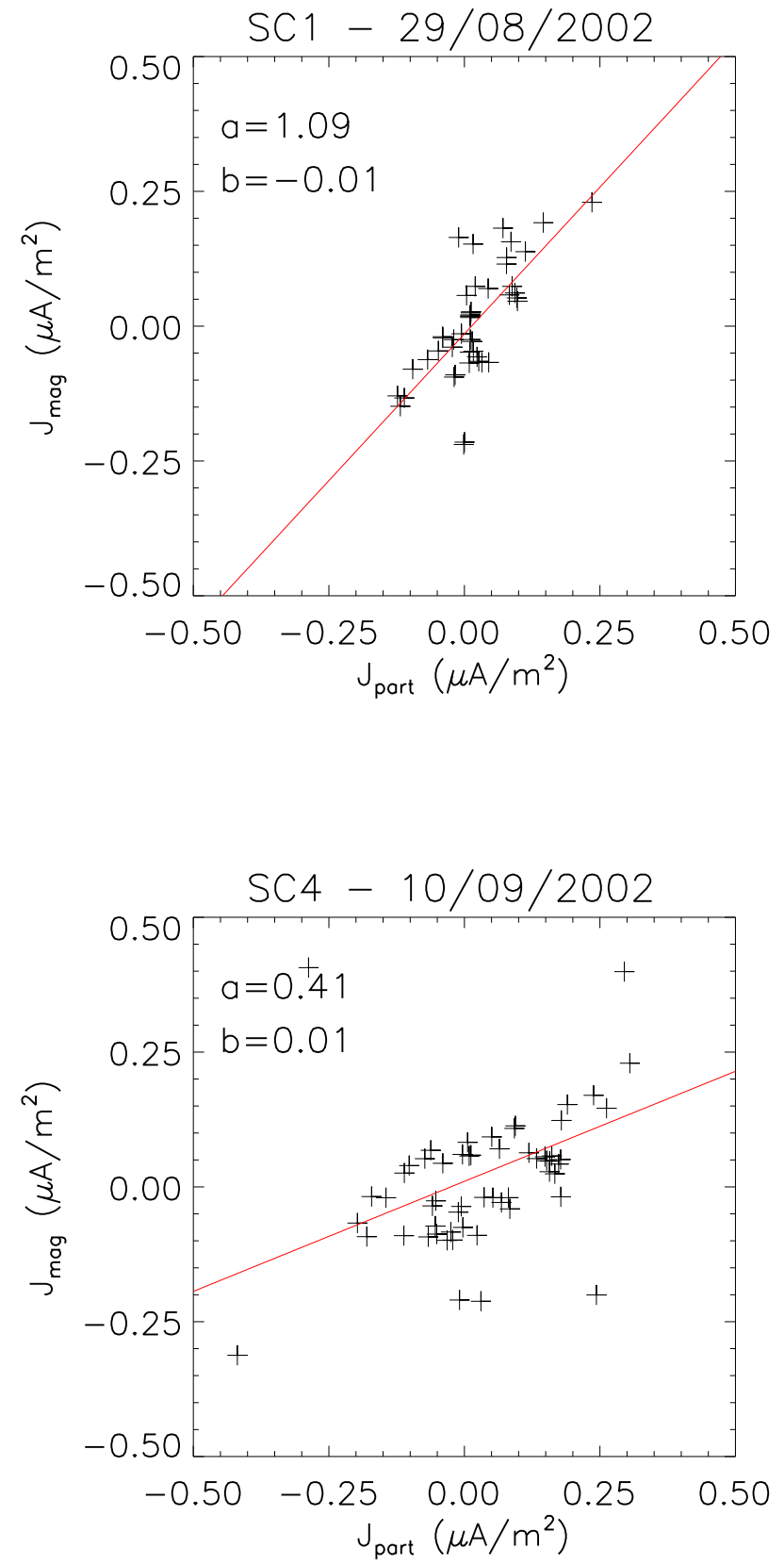

Fig. 5. Least-square linear regression fit (red line) between $J / /$ part and $J_{/ / \mathrm{mag}}$, with $a$ and $b$ : the slope and constant factor of the fit. (Top) for event A (sc-1, 29 August 2002), 16:06-16:15 UT. (Bottom) for event B (sc-4, 10 September 2002), 14:07-14:16 UT.

In order to assess more quantitatively the differences between $J_{/ / \text {part }}$ and $J_{/ / \mathrm{mag}}$, we calculate the least-square linear regression fit between the two data sets. We use only data points for which the variance ratio of the magnetic field is below 0.25 , in order to remove the more tube-like structures. This leaves between 45 and 60 pairs of data points, depending upon the event, which allows a determination of a confi- 
dent fit between the two data sets. Figure 5 shows the linear fit for the 2 events. For event A (top panel of Fig. 5), the ratio $J_{/ / \mathrm{mag}} / J_{/ / \text {part }}$ is 1.09 with a nice distribution of points along the regression line, as confirmed by a correlation coefficient of 0.71 . We have chosen to eliminate a short period (16:10:30-16:10:55 UT), where the disagreement is obvious and which could be explained by the strong concurrent IMF$B_{z}$ inversion. For event B (bottom panel of Fig. 5), the same ratio $J_{/ / \mathrm{mag}} / J_{/ / \text {part }}$ is smaller, around 0.41 , with a more dispersed distribution of points as shown by a correlation coefficient of 0.39 only. Again, we have chosen to eliminate the LLBL/cusp sub-interval (14:07:55-14:09:05 UT) where the disagreement is obvious, despite a low variance ratio and a stable polarisation angle. We will discuss the possible source of this discrepancy in the next section. It is important to note the absence of offset between the two data sets, as indicated by the very small constant coefficient of the linear regression. From the above regressions, we can state that the sign of the two FACs is statistically consistent. In terms of amplitude, the particle current is of the same order as the magnetic current for event $\mathrm{A}$ which is a very satisfactory result and larger than the magnetic current for event $B$ by a factor $\sim 2.4$. This discrepancy for event $B$ does not fit into measurement errors and an explanation will be suggested below.

\subsection{Why do particle and magnetic currents differ?}

In this section, we discuss two possible explanations to the difference between the particle and magnetic current densities: the presence of Alfvén waves and the motion of the current sheets.

Alfvén waves can be a source of transverse magnetic fluctuations in addition to static parallel currents. When relation (2) which is valid only for static structures is used for a wave of frequency $\omega$ and transverse wave number $k_{\perp}$, time variations are interpreted as spatial, which leads to a current $j_{/ / \text {static }}=j_{/ / \text {Alvén }}\left(1-\omega / k_{\perp} V_{\perp}\right)$ different from the real current $j_{/ / \text {Afruen }}$. However, in a discussion of the origin of magnetic fluctuations at auroral ionospheric altitudes, and based on the analysis of their correlation with electric measurements, Ishii et al. (1992) have shown that Alfvén waves dominate the magnetic spectrum only at high frequency (periods $<6 \mathrm{~s}$ ). These short periods are smoothed out in our analysis which averages the parallel current over $20 \mathrm{~s}$. This rules out an explanation of the observed discrepancies in terms of Alfvén waves.

In addition to being static, the parallel current structures have been assumed stationary in the Earth reference frame. This assumption, which is valid at ionospheric altitudes, is most probably no longer satisfied at the altitude of our observations. In the vicinity of its perigee, the Cluster velocity $\left(\sim 4 \mathrm{~km} . \mathrm{s}^{-1}\right)$ may be of the same order as (or even smaller than) the convection velocity which is most likely to represent the drift velocity of the current structures, either the tubes or the sheets. From relation (2), a larger (smaller) ve- locity of the current structure with respect to the observer will reduce (increase) the magnetic current density. Because the slope of the regression line between the two evaluations of the current is unity for event A (top panel of Fig. 5), the effect of the sheet motion is negligible. The sheet velocity effect is best illustrated for event B. During the period 14:07:5514:09:05 UT, it is interesting to observe that the magnetic current changes sign simultaneously with a sharp step in the convection velocity measured by CIS which occurs at the interface between the LLBL and the cusp. A northward velocity of $5 \mathrm{~km} . \mathrm{s}^{-1}$ would be sufficient to adjust the magnetic to the particle current in this LLBL/cusp region, both in amplitude and in sign. After the convection step, in the cusp region, corresponding to the correlation of the bottom panel of Fig. 5, a constant northward (parallel to the satellite orbit) component of the sheet velocity of $2.4 \mathrm{~km} . \mathrm{s}^{-1}$ can explain the systematically smaller magnetic current density. In addition, larger spatial velocity variations along the orbit can be the source of the larger variance in the regression line in event $\mathrm{B}$ than in event $\mathrm{A}$.

\section{Conclusions}

We have determined field-aligned currents from magnetic field and from particle flux measurements during two crossings of the mid-altitude polar cusp by Cluster. Magnetosheath plasma injections correlate well with the most intense pairs of field-aligned currents. By analysing the polarisation of the magnetic field, we have shown that at medium transverse scales $(80 \mathrm{~km})$, the structure of the currents is more often tube-like than sheet-like, and that current sheets are not systematically elongated in the east-west direction. The analysis of particle currents has shown that the parallel current is mainly carried by electrons. In the plane current sheets, the quantitative comparison between the two determinations of the parallel current density shows that the magnetic current is of the same order or smaller than the particle current. We suggest that current sheet motions are the source of this discrepancy.

Acknowledgements. The authors thank D. Fontaine for useful discussions. The authors thank the PIs H. Rème and E. Lucek for providing the CIS and the FGM Cluster data respectively. The authors thank also P. Décréau (PI of the WHISPER experiment) and $\mathrm{X}$. Vallières for providing the electron density deduced from the WHISPER data. The authors acknowledge the PIs N. Ness and D. J. McComas, and the ACE Science Center for providing the ACE data.

Topical Editor I. A. Daglis thanks J. Wild and P. E. Sandholt for their help in evaluating this paper.

\section{References}

Balogh, A., Carr, C. M., Acuna, M. H., Dunlop, M. W., Beek, T. J., Brown, P., Fornaçon, K.-H., Georgescu, E., Glassmeier, K.- 
H., Harris, J., Musmann, G., Oddy, T., and Schwingenschuh, K.: The CLUSTER Magnetic Field Investigation: overview of inflight performance and initial results, Ann. Geophys., 19, 12071217, 2001, http://www.ann-geophys.net/19/1207/2001/.

Berko, F. W., Hoffman, R. A., Burton, R. K., and Holzer, R. E.: Simultaneous particle and field observations of field-aligned currents, J. Geophys. Res., 80, 37-46, 1975.

Berthelier, J.-J., Cerisier, J.-C., Machard, C., Berthelier, A., and Bosqued, J.-M.: ULF electromagnetic turbulence in the high latitude topside ionosphere, J. Geophys. Res., 93, 5701-5712, 1988.

Burch, J. L., Reiff, P. H., and Sugiura, M.: Upward electron beams measured by DE-1: a primary source of dayside region-1 birkeland currents, Geophys. Res. Lett., 10, 753-756, 1983.

Burch, J. L., Menetti, J. D., and Slavin, J. A.: Dayside auroral particle acceleration mechanisms derived from Dynamics Explorer data, J. Geomag. Geoelectr., 42, 1365-1378, 1990.

Burke, W. J., Electric fields and currents observed by S3-2 in the vicinity of discrete arcs, Geophys. Monogr. Ser., 28, edited by: T. A. Potemra, 294-303, AGU, Washington, D. C., 1984.

Elphic, R. C., Bonnell, J. W., Strangeway, R. J., Keko, L., Ergun, R. E., McFadden, J. P., Carlson, C. W., Peria, W., Cattell, C. A., Klumpar, D., Shelley, E., Peterson, W., Moebius, E., Kistler, L., and Pfaff, R.: The auroral current circuit and field-aligned currents observed by FAST, Geophys. Res. Lett., 25, 2033-2036, 1998.

Erlandson, R. E., Zanetti, L. J., Potemra, T. A., Bythrow, P. F., and Lundin, R.: IMF B(y) dependence of region 1 Birkeland currents near noon, J. Geophys. Res., 93, 9804-9814, 1988.

Escoubet, C. P., Smith, M. F., Fung, S. F., Anderson, P. C., Hoffman, R. A., Basinska, E. M., and Bosqued, J.-M.: Staircase ion signature in the polar cusp-A case study, Geophys. Res. Lett., 19, 1735-1738, 1992.

Fung, S. F., and Hoffman, R. A.: Finite geometry effects of fieldaligned currents, J. Geophys. Res., 97, 8569-8579, 1992.

Hoffman, R. A., Sugiura, M., and Maynard, N. C.: Current carriers for the field-aligned current system, Adv. Space Res., 5, 109126, 1985.

Iijima, T., Potemra, T. A., Zanetti, L. J., and Bythrow, P. F.: Large-scale Birkeland currents in the dayside polar region during strongly northward IMF - A new Birkeland current system, J. Geophys. Res., 89, 7441-7452, 1984.

Iijima, T. and Potemra, T. A.: Field-aligned currents in the dayside cusp observed by Triad, J. Geophys. Res., 81, 5971-5979, 1976.

Ishii, M., Sugiura, M., Iyemori, T., and Slavin, J. A.: Correlation between magnetic and electric field perturbations in the fieldaligned current regions deduced from DE-2 observations, J. Geophys. Res., 97, 13 877-13 887, 1992.

Johnstone, A. D., Alsop, C., Burge, S., et al.: 'PEACE: a Plasma electron and current experiment', Space Sci. Rev., 79, 351-398, 1997.

Klumpar, D. M.: Relationships between auroral particle distributions and magnetic field perturbations associated with fieldaligned currents, J. Geophys. Res., 84, 6524-6532, 1979.

Klumpar, D. M., Burrows, J. R., and Wilson, M. D.: Simultaneous observations of field-aligned currents and particle fluxes, Geophys. Res. Lett., 3, 395-398, 1976.

Lockwood, M., Milan, S. E., Onsager, T., Perry, C. H., Scudder, J. A., Russell, C. T., and Brittnacher, M.: Cusp ion steps, field- aligned currents and poleward moving auroral forms, J. Geophys. Res., 106, 29 555-29 569, 2001.

Marchaudon, A., Cerisier, J.-C., Greenwald, R., and Sofko, G.: Electrodynamics of a FTE: Experimental test of the Southwood model, Geophys. Res. Lett., 31, doi:10.1029/2004GL019922, 2004.

McDiarmid, D. R. and McNamara, A. G.: Radio aurora in the dayside auroral oval spatial relationships with field-aligned currents and energetic particles, J. Geophys. Res., 83, 3226-3234, 1978.

Oksavik, K., Søraas, F., Moen, J., Pfaff, R., Davies, J. A., and Lester, M.: Simultaneous optical, CUTLAS HF radar, and FAST spacecraft observations: signatures of boundary layer processes in the cusp, Ann. Geophys., 22, 511-525, 2004, http://www.ann-geophys.net/22/511/2004/.

Potemra, T. A.: Field-aligned (Birkeland) currents, Space Sci. Rev., 42, 295-311, 1985.

Potemra, T. A., Peterson, W. K., Doering, J. P., Bostrom, C. O. McEntire, R. W., and Hoffman, R. A.: Low-energy particle observations in the quiet dayside cusp from AE-C and AE-D, J. Geophys. Res., 82, 4765-4776, 1977.

Reiff, P. H., Burch, J. L., and Hill, T. W.: Solar wind plasma injections at the dayside magnetospheric cusp, J. Geophys. Res., 82, 479-491, 1977.

Rème H., Aoustin, C., Bosqued, J.-M., et al.: First multispacecraft ion measurements in and near the Earth's magnetosphere with the identical Cluster ion spectrometry (CIS) experiment, Ann. Geophys., 19, 1303-1354, 2001, http://www.ann-geophys.net/19/1303/2001/.

Robert, P. R., Gendrin, R., Perraut, S., Roux, A., and Pedersen, A.: GEOS-2 identification of rapidly moving current structures in the equatorial outer magnetosphere, J. Geophys. Res., 89, 819-840, 1984.

Sandholt, P. E., Farrugia, C. J., and Denig, W. F.: Dayside aurora and the role of IMF $|\mathrm{By} / \mathrm{Bz}|$ : detailed morphology and response to magnetopause reconnection, Ann. Geophys., 22, 613-628, 2004a.

Sandholt, P. E., Farrugia, C. J., and Denig, W. F.: Detailed dayside auroral morphology as a function of local time for southeast IMF orientation: implications for solar wind - magnetosphere coupling, Ann. Geophys., 22, 3537-3560, 2004b.

Seran, E., and Cerisier, J.-C.: Current layers: Influence of the finite size and non-uniform current distribution, J. Atmos. SolarTerrestr. Phys., 67, 729-737, 2005.

Southwood, D. J.: The ionospheric signature of flux transfer events, J. Geophys. Res., 92, 3207-3213, 1987.

Sugiura, M., Iyemori, T., Hoffman, R. A., Maynard, N. C., Burch, J. L., and Winningham, J. D.: Relationships between field-aligned currents, electric fields, and particle precipitation as observed by Dynamics Explorer-2, in: Magnetospheric Currents, Geophys. Monogr. Ser., 28, edited by: Thomas, A., Potemra, 96-103, AGU, Washington, D. C., 1984.

Theile, B. and Wilhelm, K.: Field-aligned currents above an auroral arc, Planet. Space Sci., 28, 351-355, 1980.

Yamauchi, M., Lundin, R., Eliasson L., Ohtani, S., and Clemmons, J. H.: Relationships between large-, meso-, and small-scale fieldaligned currents and their current carriers, in Polar Cap Boundary Phenomena, edited by: J. Moen et al., 173-188, Kluwer Academic Publishers, Netherlands, 1998. 